\title{
Psychometric Properties of the Hypomania Checklist-32 in Korean Patients with Mood Disorders
}

\author{
Bo-Hyun Yoon ${ }^{1}$, Jules Angst ${ }^{2}$, Won-Myong Bahk ${ }^{3}$, Hee Ryung Wang ${ }^{3}$, Seung-Oh Bae ${ }^{4}$, Moon-Doo Kim ${ }^{5}$, \\ Young-Eun Jung, Kyung Joon Min ${ }^{6}$, Hwang-Bin Lee ${ }^{7}$, Seunghee Won ${ }^{8}$, Jeongwan Hong ${ }^{9}$, Myong Su Choi ${ }^{10}$, \\ Duk-In Jon ${ }^{11}$, Young Sup Woo ${ }^{3}$ \\ Department of Psychiatry, Naju National Hospital, Naju, Korea, ${ }^{2}$ Department of Psychiatry, Psychotherapy and Psychosomatics, Psychiatric \\ Hospital, University of Zurich, Zurich, Switzerland, ${ }^{3}$ Department of Psychiatry, College of Medicine, The Catholic University of Korea, Seoul, \\ ${ }^{4}$ Hangang Mental Clinic, Gimpo, ${ }^{5}$ Department of Psychiatry, Jeju National University Hospital, Jeju, ${ }^{6}$ Department of Psychiatry, Chung-Ang \\ University College of Medicine, Seoul, ${ }^{7}$ Department of Psychiatry, National Center for Mental Health, Seoul, ${ }^{8}$ Department of Psychiatry, Kyungpook \\ National University School of Medicine, Daegu, ${ }^{9}$ Department of Psychiatry, Iksan Hospital, Iksan, ${ }^{10}$ Choimyongsu Psychiatric Clinic, Jeonju, \\ ${ }^{11}$ Department of Psychiatry, Hallym University Sacred Heart Hospital, Hallym University College of Medicine, Anyang, Korea
}

Objective: The aim of this study was to examine the validity of the Korean version of the Hypomania Checklist-32, second revision (HCL-32-R2) in mood disorder patients.

Methods: A total of 454 patients who diagnosed as mood disorder according to Structured Clinical Interview for DSM-IV Axis | Disorders, clinician version (SCID-CV) (bipolar disorder [BD] I, n=190; BD-||, n=72; and major depressive disorder [MDD], n=192) completed the Korean module of the HCL-32-R2 (KHCL-32-R2).

Results: The KHCL-32-R2 showed a three-factorial structure (eigenvalue $>2$ ) that accounted for $43,26 \%$ of the total variance Factor 1 was labeled "active/elated" and included 16 items; factor 2, "irritable/distractible" and included 9 items; and factor 3 was labeled "risk-taking/indulging" and included 9 items. A score of 16 or more on the KHCL-32-R2 total scale score distinguished between $\mathrm{BD}$ and MDD, which yielded a sensitivity of $70 \%$ and a specificity of $70 \%$. MDD and BD-\|l also could be differentiated at a cut-off of 15 with maximized sensitivity (0.67) and specificity (0.66). Cronbach's alpha of KHCL-32-R2 and its subsets (factors 1, 2, and 3) were 0.91, 0.89, 0.81 and 0.79, respectively. Correlations between KHCL-32-R2 and MontgomeryAsberg Depression Rating Scale, Young Mania Rating Scale and Korean version of Mood Disorder Questionnaire were - 0.66 $(p=0.41),-0.14(p=0.9)$, and $0.61 \quad(p<0.001)$, respectively.

Conclusion: The KHCL-32-R2 may be a useful tool in distinguishing between bipolar and depressive patients in clinical settings.

KEY WORDS: HCL-32-R2; Validation; Sensitivity; Specificity; Bipolar disorder; Major depression.

\section{INTRODUCTION}

Bipolar disorder (BD) is a major psychiatric disorder with a highly recurrent and chronic nature. ${ }^{1)}$ It is often under- or misdiagnosed, thus delaying the administration of effective treatment, with one- to two-thirds of patients with $\mathrm{BD}$ not receiving appropriate treatment due to misdiagnosis. ${ }^{2)} \mathrm{BD}$, particularly BD-II, is frequently misdiagnosed as major depressive disorder (MDD) in clinical

\footnotetext{
Received: January 10, 2017 / Revised: February 7, 2017

Accepted: February 16, 2017

Address for correspondence: Won-Myong Bahk, MD, PhD Department of Psychiatry, Yeouido St. Mary's Hospital, College of Medicine, The Catholic University of Korea, 10 63-ro, Yeongdeungpo-gu, Seoul 07345, Korea

Tel: +82-2-3779-1250, Fax: +82-2-780-6577

E-mail:wmbahk@catholic.ac.kr
}

practice. ${ }^{3)}$ Hypomania as an element of BD-II is often not experienced or not recognized by the patient and their families as pathological; therefore, it is usually not reported to doctors and remains under-diagnosed in 25 to $50 \%$ of MDD patients. ${ }^{4}$ Furthermore, clinicians tend not to investigate hypomania if patients present with a depressive episode. ${ }^{4)}$ All of these factors contribute to failure to accurately diagnose $\mathrm{BD}$ in clinical practice. Recommendations for improving the diagnostic accuracy of BD include establishing a comprehensive history of hypomania/mania, and supplementing it with the administration of screening tools. ${ }^{5,6)}$ Diagnostic error has also often stemmed from the misinterpretation of hypomania as the result of antidepressant treatment ("drug induced switch to hypomania"); it is now agreed that patients who switch are true cases of BD-II, now also recognized in Diagnostic

(c) This is an Open-Access article distributed under the terms of the Creative Commons Attribution Non-Commercial License (http://creativecommons.org/licenses/by-nc/4.0) which permits unrestricted non-commercial use, distribution, and reproduction in any medium, provided the original work is properly cited. 
and Statistical Manual of Mental Disorders 5th edition (DSM-5). ${ }^{7}$ Of course, such over-diagnosis of MDD decreases the specificity of screening instruments for hypomania.

Standardized structured diagnostic interviews such as the Mini International Neuropsychiatric Interview (MINI) ${ }^{8}$ and the Structural Clinical Interview for DSM-IV (SCID) ${ }^{9}$ are often used in research and even clinical practice, but applying these tools is time-consuming and requires well-trained raters. Recently, several self-report screening tools have been developed to overcome these difficulties and to aid in the detection of BD. Based on DSM-IV diagnostic criteria, the 32-item Hypomania Checklist (HCL32) ${ }^{4)}$ Mood Disorder Questionnaire (MDQ), ${ }^{10)}$ and Bipolar Spectrum Diagnostic Scale ${ }^{11)}$ are instruments intended for widespread screening of bipolarity in patient with mood disorders.

Initially developed as a 32-question instrument (HCL$32-\mathrm{R} 1$ ), in its recently modified version, the HCL-32 includes an additional 2 questions (HCL-32-R2). ${ }^{12}$ Transcultural analysis of the BRIDGE Study (Bipolar Disorders: Improving Diagnosis, Guidance and Education), which administered the HCL-32-R2, showed that it had good stability across five geographical regions (Iberia, Central Europe, Eastern Europe, North Africa/the Near East, and the Far East including Korea). ${ }^{13)}$

Although the Korean version of the HCL-32-R1 has been validated, ${ }^{14,15)}$ the study of the psychometric properties of the HCL-32-R2 has not yet been tested in Korea. Therefore, the aim of this study was to exam the validity of the Korean adaptation of the HCL-32-R2 (KHCL-32-R2).

\section{METHODS}

\section{Subjects}

This study was carried out from September 1, 2013 to March 31, 2015 via 10 universities or psychiatric hospitals in Korea.

Mood disorders patients who were diagnosed with BDs (BD-I, BD-II, or BD-not otherwise specified [NOS]) or unipolar disorders (MDD, depressive disorder NOS [DEP-NOS], or dysthymic disorder [DD]) via the SCID were recruited. Their ages ranged between 18 and 60 years and all had a minimum of six years of education. One main eligibility criteria for participation in this study was the ability to provide independent written informed consent, regardless of their symptom severity. Exclusion criteria included mood disorders secondary to general medical or neurological conditions, patients diagnosed with unstable or severe clinical status, those who could not cooperate with the study procedures, patients who received eletroconvulsive therapy or modified eletroconvulsive therapy during the previous four weeks, and individuals who were illiterate or suffered from mental retardation, dementia or intellectual impairment. The study was approved by the relevant ethics committees or institutional review boards.

A total of 454 patients were enrolled; the total number of enrolled BD-I, BD-II and MDD patients was 190 (41.9\%), 72 (15.9\%) and $192(42.3 \%)$, respectively. Two hundred seventy-six patients $(60.8 \%)$ were female and $324(71.4 \%)$ were outpatients.

\section{Instruments and Assessment Procedure}

Patients with mood disorders were referred to the research team at each recruitment site to be screened for eligibility. All inpatients and patients who visited outpatient clinic and fulfilled the study criteria were invited to participate in the study.

The Montgomery-Asberg Depression Rating Scale (MADRS $)^{16,17)}$ was used to measure the severity of depressive symptoms within the past week. The Young Mania Rating Scale (YMRS) was also applied to all patients. ${ }^{18,19)}$ The MDQ, a self-report questionnaire used to screen mania/hypomania, was administered. ${ }^{10)}$ It consisted of 13 yes/no questions, reflecting DSM-IV inclusion criteria, followed by a single yes/no question about whether the symptoms clustered simultaneously and another question about the causality of the symptoms. The Korean version of MDQ was used in this study. ${ }^{20)}$

The HCL-32-R2 is a slightly extended version of the original 32-item HCL-32 scale (R1). ${ }^{4,21)}$ Compared to the HCL-32-R1, ${ }^{4)}$ the HCL-32-R2 includes two additional items ("I gamble more" and "I eat more"), yielding a total of 34 items. The HCL-32-R2 used in this study was adopted from the Korean HCL-32-R2 module included in BRIDGE study ${ }^{12)}$ with the author's permission. The Korean version of the HCL-32-R1 has been validated, ${ }^{14,15)}$ but the extended version has not yet been validated.

The diagnostic assessment of $\mathrm{BD}$ and MDD was conducted at the time of inclusion with the SCID clinical version (SCID-CV) to establish DSM-IV diagnoses by clinicians who were blind to the diagnosis and the HCL-32-R2 scores of each subject. ${ }^{9)}$

After providing written informed consent, patients were invited to complete the HCL-32-R2, MDQ, and the additional clinical assessments including MADRS and YMRS. Sociodemographic information was collected 
from medical records and patients' families.

\section{Statistical Analysis}

Data distribution was ascertained using the KolmogorovSmirnov test. Parametric and non-parametric tests included (1) the chi-square or Mann-Whitney $U$ test to compare frequency distributions and (2) the one-way ANOVA (using least significant difference post-hoc method) or Kruskal-Wallis tests to compare continuous outcomes, respectively.

Optimal cut-off scores maximizing the sensitivity (proportion of patients with a SCID diagnosis of BD who screened positive on the HCL-32-R2) and specificity (proportion of patients not meeting SCID criteria for BD who screened negative on the HCL-32-R2) were calculated for the KHCL-32-R2. These optimal cut-off scores were determined in order to discriminate between MDD and BD, between MDD and BD-I, and between MDD and BD-II. The area under the receiver operating characteristic (ROC) curve (AUC) was calculated at various cut-off points. The sensitivity and specificity at various cut-offs were presented in figures and tables. If the Cronbach's alpha was greater than 0.7 , it could be concluded that the internal consistency of the KHCL-32-R2 was acceptable. ${ }^{22)}$

The dimensionality of the KHCL-32-R2 was measured by exploratory factor analysis. Principal component analysis was carried out to extract the factors; varimax rotation was used to obtain the most meaningful original factor structure of the KHCL-32-R2. Items were assigned to a specific factor when loadings were at least 0.4. Determination of the number of factors to retain was based on several criteria: Kaiser's criterion (factors with eigenvalue $>1.0$ ), the scree plot, and Horn's parallel test, but principally this decision was based on the coherence and interpretability of factors.

The association between the current mood state measured with the KHCL-32-R2 current mood state item, score on the MADRS, YMRS, MDQ and KHCL-32-R2 were computed by Spearman's and Pearson's correlations. Data were analyzed with the IBM SPSS statistics, version 22.0 (IBM Co., Armonk, NY, USA). The level of significance was $p<0.05$ (two tailed) using $95 \%$ confidence intervals (CIs) across analyses.

\section{RESULTS}

\section{Demographic Characteristics of Subjects}

The population-based equivalent subject number was provided by all participating centers. All 514 patients were screened and invited to participate in the study; 60 patients (11.7\%) did not participate due to the following reasons: declined to participate, failure to complete interview, or violation of study procedure and study protocol. A final total of 454 patients were included in analysis: 192 diagnosed with MDD (42.3\%), 190 diagnosed with BD-I (41.9\%), and 72 diagnosed with BD-II (15.9\%). Essential demographic and clinical data of subjects have been outlined in Table 1. Patients with BD-I were younger, more educated, less likely to be married, more frequently admitted to the hospital, and had an earlier age at onset of illness than those with MDD. Patients with BD-I also had significantly higher YMRS scores, lower MADRS scores and higher MDQ total scores. Among the three groups, depressive episodes were more frequent in BD-II patients. MDQ and KHCL-32-R2 scores were higher in BD patients than in MDD patients.

Table 1. Demographic and clinical characteristics of patients

\begin{tabular}{|c|c|c|c|c|c|}
\hline & Total $(n=454)$ & $\operatorname{MDD}(n=192)$ & BD-I $(n=190)$ & $B D-\|(n=72)$ & $p$ value \\
\hline Sex, male & $178(39.2)$ & $66(34.4)$ & $86(45.3)$ & $26(36.1)$ & NS \\
\hline Age (yr) & $40.61 \pm 13.13$ & $42.95 \pm 14.04$ & $38.60 \pm 12.08$ & $39.69 \pm 12.47$ & $<0.001(\mathrm{BD}-\mathrm{I}<\mathrm{MDD})$ \\
\hline Education (yr) & $12.82 \pm 2.89$ & $12.40 \pm 2.78$ & $13.29 \pm 2.84$ & $12.69 \pm 3.13$ & $<0.05(\mathrm{BD}-\mathrm{I}>\mathrm{MDD})$ \\
\hline Married & $200(44.1)$ & $104(54.2)$ & $64(33.7)$ & $32(44.4)$ & $<0.001$ \\
\hline Outpatient & $324(71.7)$ & $160(84.2)$ & $104(54.7)$ & $60(83.3)$ & $<0.001$ \\
\hline Age at onset $(y r)$ & $31.10 \pm 12.63$ & $37.03 \pm 13.15$ & $26.65 \pm 10.37$ & $27.06 \pm 10.08$ & $<0.001(\mathrm{MDD}>\mathrm{BD}-\mathrm{I}=\mathrm{BD}-\mathrm{II})$ \\
\hline Number of depressive episodes & $2.90 \pm 3.61$ & $2.39 \pm 1.98$ & $2.29 \pm 2.32$ & $5.72 \pm 6.84$ & $<0.001$ (BD-II $>$ BD-I, MDD) \\
\hline Number of admission & $2.03 \pm 3.61$ & $0.21 \pm 0.46$ & $5.47 \pm 5.43$ & $0.10 \pm 0.30$ & $<0.001$ (BD-I>BD-II, MDD) \\
\hline YMRS, total & $7.40 \pm 10.28$ & $3.80 \pm 3.56$ & $11.84 \pm 13.99$ & $5.25 \pm 5.15$ & $<0.001(\mathrm{BD}-\mathrm{I}>\mathrm{BD}-\mathrm{II}, \mathrm{MDD})$ \\
\hline MADRS, total & $15.62 \pm 11.21$ & $19.51 \pm 10.08$ & $10.14 \pm 9.26$ & $19.83 \pm 12.68$ & $<0.001(\mathrm{BD}-\mathrm{I}<\mathrm{BD}-\mathrm{II}, \mathrm{MDD})$ \\
\hline$M D Q$, total & $7.35 \pm 3.93$ & $5.25 \pm 3.77$ & $8.77 \pm 3.40$ & $9.19 \pm 2.93$ & $<0.001(\mathrm{BD}-\mathrm{I}, \mathrm{BD}-\mathrm{II}>\mathrm{MDD})$ \\
\hline KHCL-32-R2, total & $16.25 \pm 8.01$ & $12.73 \pm 8.21$ & $18.89 \pm 6.86$ & $18.64 \pm 6.71$ & $<0.001(\mathrm{BD}-\mathrm{I}, \mathrm{BD}-\mathrm{II}>\mathrm{MDD})$ \\
\hline
\end{tabular}

Values are presented as number (\%) or mean \pm standard deviation.

MDD, major depression; BD-I, bipolar I disorder; BD-II, bipolar II disorder; YMRS, Young Mania Rating Scale; MADRS, Montgomery-Asberg Depression Rating Scale; MDQ, Mood Disorders Questionnaire; KHCL-32-R2, the Korean adaptation of Hypomania Checklist-32, second version; NS, not significant. 


\section{Frequency of Positive Responses in the KHCL-32-R2 according to MDD and BD Diagnosis}

Figure 1 shows the frequency of positive responses (a score of 1 or more for each item) for patients diagnosed with MDD and BD on items in the KHCL-32-R2. Differences in positive responses between patients with $\mathrm{BD}$ and patients with MDD were found to be significant; patients with $\mathrm{BD}$ reported more positive responses than patients with MDD $(p<0.001)$, with the exception of items 7,13 , $31,32,33$ and 34 .

\section{Current Mood State, Symptom Measures and KHCL-32-R2 Scores}

Table 2 shows that there was a significant difference in mean total KHCL-32-R2 scores between the seven levels of current mood states in patients with MDD $(p<0.05)$; however, patients with BD did not show significant differ-

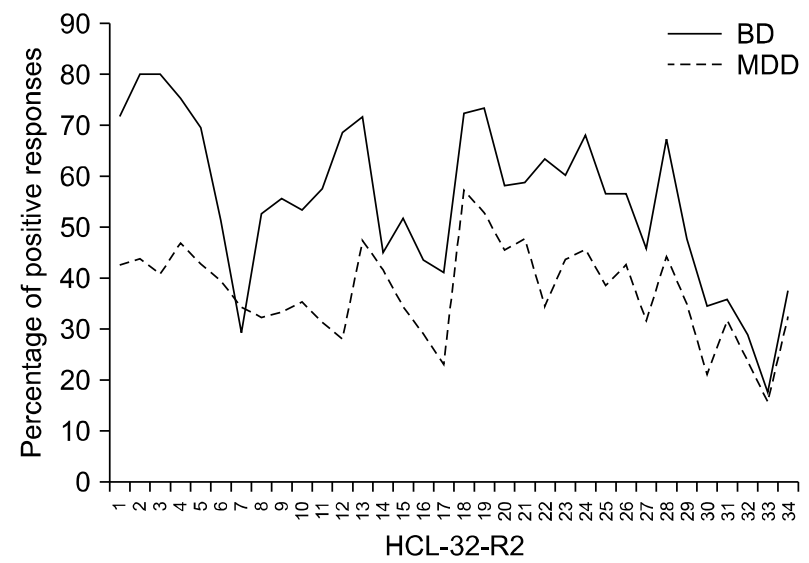

Fig. 1. Frequency of positive responses of Hypomania Check List-32 (HCL-32) items between bipolar disorder (BD) and major depressive disorder (MDD) patients (total=494, BD=262, MDD=192). HCL32-R2, HCL-32 second revision. There were significant differences between the two groups in all items $(p<0.001)$ except items 7 , $14,31,32,33$ and 34 . ences at different levels of current mood states. There were no significant correlations between current mood state and KHCL-32-R2 scores in patients with MDD (rho $=0.06, p=0.84)$ or $\mathrm{BD}(\mathrm{rho}=-0.2, p=0.65)$.

In order to examine the relationship between symptom measures and KHCL-32-R2 scores, total scores of MADRS, YMRS, and MDQ scores were compared using Pearson's and Spearman's correlation. Total scores of MADRS $(\mathrm{rho}=-0.07, p=0.36)$ and YMRS (rho $=-0.10, p=0.20)$ were not significantly correlated with KHCL-32-R2 score; MDQ scores were significantly correlated with KHCL-32-R2 score $(\mathrm{r}=0.73, p<0.001)$.

\section{Factor Analysis of HCL-32-R2}

Principal component analysis with varimax rotation revealed that the eigenvalue for eight factors was greater than 1 , accounting for $60.03 \%$ of the total variance. The screeplot showed that would justify retaining 3 components, of which eigenvalues were above 2 (Fig. 2). The ei-

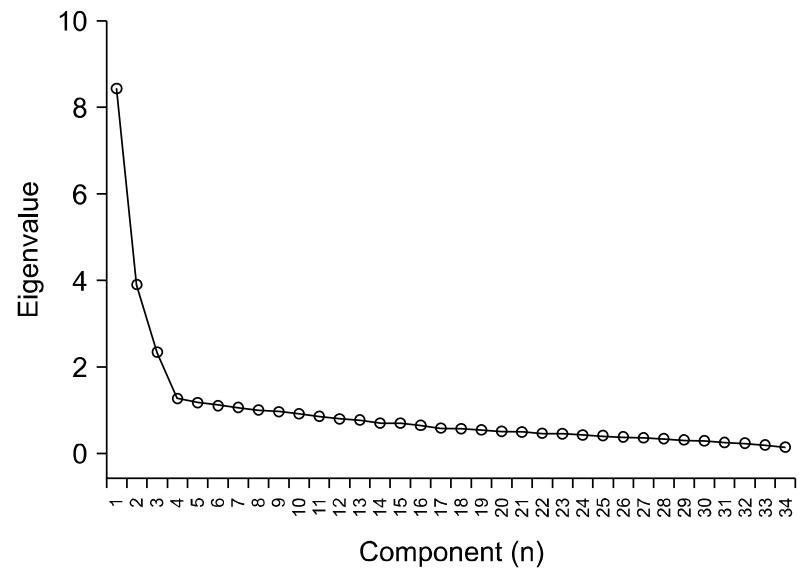

Fig. 2. Scree plot of Hypomania Check List-32 second revision at exploratory factor analysis. The three factors were retained (eigenvalue $>2$ ).

Table 2. KHCL-32-R2 scores at different level of current mood state

\begin{tabular}{lcccc}
\hline \multirow{2}{*}{ Current mood state } & \multicolumn{2}{c}{ MDD $(n=192)$} & \multicolumn{2}{c}{ BD $(n=262)$} \\
\cline { 2 - 5 } \cline { 4 - 5 } & Patient $(n)$ & Score (mean \pm SD) & Patient $(n)$ & Score (mean \pm SD) \\
\hline 1. Much worse than usual & 16 & $12.00 \pm 0.87$ & 22 & $19.64 \pm 5.10$ \\
2. Worse than usual & 22 & $13.27 \pm 9.43$ & 20 & $20.70 \pm 4.47$ \\
3. A little worse than usual & 40 & $15.60 \pm 9.45$ & 38 & $19.26 \pm 7.11$ \\
4. Neither better nor worse than usual & 96 & $10.60 \pm 0.48$ & 130 & $17.75 \pm 7.32$ \\
5. A little better than usual & 10 & $17.00 \pm 10.89$ & 22 & $18.27 \pm 7.95$ \\
6. Better than usual & 8 & $18.50 \pm 8.77$ & 24 & $21.75 \pm 4.85$ \\
7. Much better than usual & 0 & & 6 & $20.33 \pm 3.39$ \\
P value* & & $\mathbf{0 . 0 2 1}$ & & $\mathbf{0 . 2 3 5}$ \\
\hline
\end{tabular}

KHCL-32-R2, the Korean adaptation of Hypomania Checklist-32, second version; MDD, major depressive disorder; BD, bipolar disorders; SD, standard deviation.

${ }^{*}$ By Kruskal-Wallis test. 
genvalue for factor 1, 2, and 3 were 8.44, 3.92, and 2.35, respectively, while other factors had eigenvalues less than 2. The first three factors accounted for $45.46 \%$ of the total variance (Table 3 ). If all items suppressed absolute factor loadings less than 0.4 and deleted when cross-loaded on more than two factors, 5 items would be excluded from HCL-32-R2. However, clinical decision of coherence made all items included to the analysis. Factor analysis showed that the first three factors accounted for $43.26 \%$ of the variance. When forced two-factor solution was conducted according to previous reports, ${ }^{23)}$ four items were excluded and found to account for $36.36 \%$ of the variance. Although many previous reports have adapted a two-factor solution, we chose to use a three-factor solution retain- ing the whole 34 items in the present study because statistical tests (Kaiser's criterion, scree test, and Horn's parallel test) showed that three-factor solution had fewer unassigned items and clearer interpretation than two-factor solution (Table 3).

Factor 1 comprised 16 items (items 2-6, 9-15, 18, 20, 22 and 28) and could be described as "active/elated". Factor 2 comprised 9 items (items 2, 8, 19,21, and 23-27) and was labeled as "irritable/distractible'. Factor 3 included 9 items (items 7, 16, 17, and 29-34) and was named "risktaking/indulging". Factors 2 and 3 could be characterized as the "dark side" of mania, as Angst et al..$^{4)}$ originally described it (Table 3).

Table 3. Item loadings for the three factors of the KHCL-32-R2 from the exploratory factor analysis

\begin{tabular}{|c|c|c|c|}
\hline \multirow{2}{*}{ Item description } & \multicolumn{3}{|c|}{ Factor loading } \\
\hline & Factor 1 & Factor 2 & Factor 3 \\
\hline 1. I need less sleep & & 0.57 & \\
\hline 2. I feel more energetic and more active & 0.71 & & \\
\hline 3. I am more self-confident & 0.71 & & \\
\hline 4. I enjoy my work more & 0.74 & & \\
\hline 5. I am more sociable (make more phone calls, go out more) & 0.61 & & \\
\hline 6. I want to travel and do travel more & 0.41 & & \\
\hline 7. I tend to drive faster or take more risks when driving & & & 0.42 \\
\hline 8. I spend more/too much money & & 0.46 & \\
\hline 9. I take more risks in my daily life (in my work and/or other activities) & 0.43 & & \\
\hline 10. I am physically more active (sport, etc.) & 0.60 & & \\
\hline 11. I plan more activities or projects & 0.62 & & \\
\hline 12. I have more ideas, I am more creative & 0.67 & & \\
\hline 13. I am less shy or inhibited & 0.59 & & \\
\hline 14. I wear more colorful and more extravagant clothes/make-up & 0.39 & & \\
\hline 15. I want to meet or actually do meet more people & 0.53 & & \\
\hline 16. I am more interested in sex, and/or have increased sexual desire & & & 0.67 \\
\hline 17. I am more flirtatious and/or am sexually more active & & & 0.65 \\
\hline 18. I talk more & 0.52 & & \\
\hline 19. I think faster & & 0.51 & \\
\hline 20. I make more jokes or puns when I am talking & 0.50 & & \\
\hline 21. I am more easily distracted & & 0.67 & \\
\hline 22. I engage in lots of new things & 0.52 & & \\
\hline 23. My thoughts jump from topic to topic & & 0.61 & \\
\hline 24. I do think more quickly and/or more easily & & 0.46 & \\
\hline 25. I am more impatient and/or get irritable more easily & & 0.72 & \\
\hline 26. I can be exhausting or irritating for others & & 0.70 & \\
\hline 27. I get into more quarrels & & 0.55 & \\
\hline 28. My mood is higher, more optimistic & 0.69 & & \\
\hline 29. I drink more coffee & & & 0.45 \\
\hline 30. I smoke more cigarettes & & & 0.67 \\
\hline 31. I drink more alcohol & & & 0.58 \\
\hline 32. I take more drugs (sedatives, anxiolytics, stimulants) & & & 0.60 \\
\hline 33. I gamble more & & & 0.78 \\
\hline 34. I eat more & & & 0.36 \\
\hline Eigenvalue & 8.44 & 3.92 & 2.34 \\
\hline$\%$ of variance (total, $43.26 \%$ ) & 24.83 & 11.53 & 6.91 \\
\hline
\end{tabular}

KHCL-32-R2, the Korean adaptation of Hypomania Checklist-32, second version.

Two items (item 14 and item 34) were included although factor loadings <0.4. Factor 1, 'active/elated'; factor 2, 'irritable/distractible'; factor 3, 'risk-taking/indulging'. 

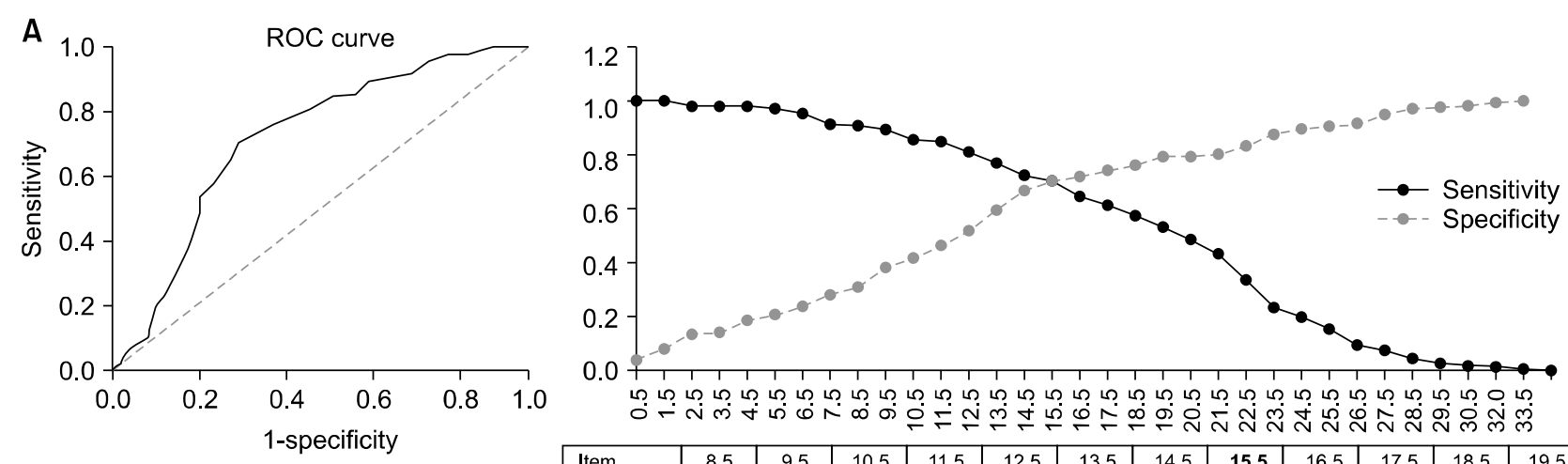

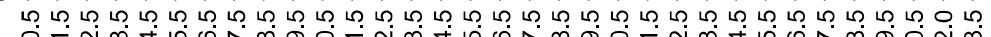

\begin{tabular}{|l|c|c|c|c|c|c|c|c|c|c|c|c|}
\hline Item & 8.5 & 9.5 & 10.5 & 11.5 & 12.5 & 13.5 & 14.5 & $\mathbf{1 5 . 5}$ & 16.5 & 17.5 & 18.5 & 19.5 \\
\hline Sensitivity & 0.908 & 0.893 & 0.855 & 0.847 & 0.809 & 0.771 & 0.725 & $\mathbf{0 . 7 0 2}$ & 0.649 & 0.611 & 0.573 & 0.534 \\
\hline Specificity & 0.312 & 0.385 & 0.417 & 0.469 & 0.521 & 0.594 & 0.667 & $\mathbf{0 . 6 9 8}$ & 0.719 & 0.74 & 0.76 & 0.792 \\
\hline
\end{tabular}
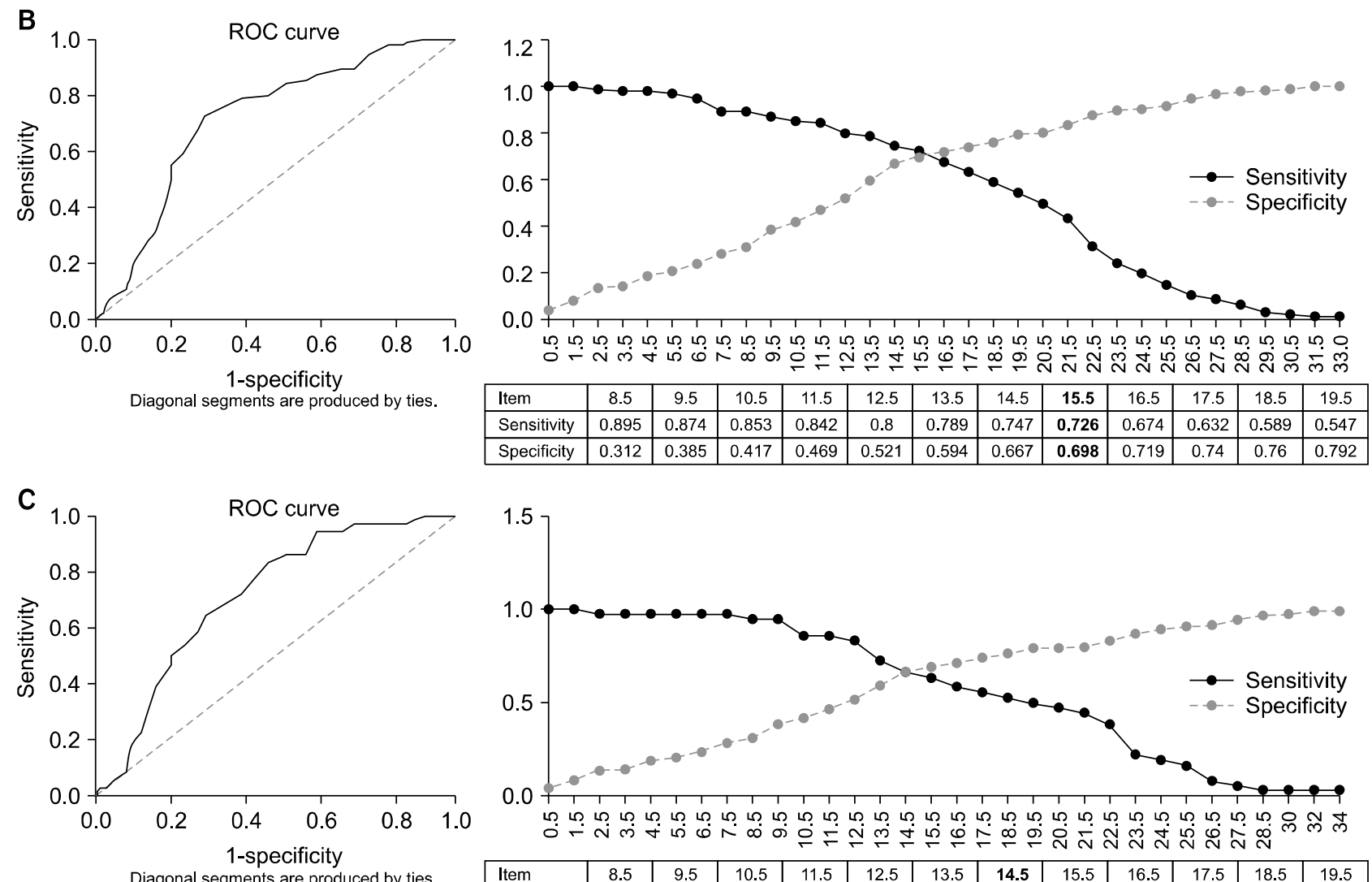

Diagonal segments are produced by ties.

\begin{tabular}{|l|c|c|c|c|c|c|c|c|c|c|c|c|}
\hline Item & 8.5 & 9.5 & 10.5 & 11.5 & 12.5 & 13.5 & $\mathbf{1 4 . 5}$ & 15.5 & 16.5 & 17.5 & 18.5 & 19.5 \\
\hline Sensitivity & 0.944 & 0.944 & 0.861 & 0.861 & 0.833 & 0.722 & $\mathbf{0 . 6 6 7}$ & 0.639 & 0.583 & 0.556 & 0.528 & 0.5 \\
\hline Specificity & 0.312 & 0.385 & 0.417 & 0.469 & 0.521 & 0.594 & $\mathbf{0 . 6 6 7}$ & 0.698 & 0.719 & 0.74 & 0.76 & 0.792 \\
\hline
\end{tabular}

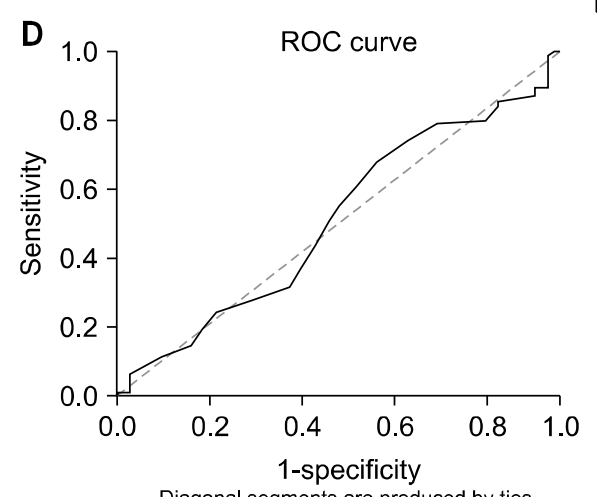

Diagonal segments are produced by ties.
Fig. 3. Sensitivity and specificity of Hypomania Checklist-32 second revision (HCL-32-R2) at various cut-offs. (A) Between $\mathrm{BD}$ and MDD. $A \cup C=0.72(95 \% \mathrm{Cl}, 0.67-0.77 ; p<0.001)$. Cut-off $=16$, sensitivity=0.70, specificity $=0.70$. (B) Between BD-I and MDD. AUC $=0.72(95 \% \mathrm{Cl}$, 0.67-0.77; $p<0.001$ ). Cut-off $=16$, sensitivity $=0.73$, specificity $=0.70$. (C) Between BD-II and MDD. AUC=0.71 (95\% Cl, 0.65-0.78; $p<$ 0.001 ). Cut-off $=15$, sensitivity $=0.667$, specificity $=0.667$. (D) Between BD-I and BD-II. AUC=0.51 (95\% Cl, 0.43-0.59; $p=0.81)$.

ROC, receiver operating characteristic; AUC, area under the curve; $\mathrm{BD}$, bipolar disorder; $\mathrm{MDD}$, major depressive disorder; $\mathrm{Cl}$, confidence interval. 


\section{Internal Consistency}

The value of Cronbach's alpha for the KHCL-32-R2 was 0.91 in the whole sample, 0.89 for factor $1,0.81$ for factor 2 and 0.79 for factor 3 .

\section{Comparison between MDD and BD KHCL-32-R2 Total and Subscale Scores}

The mean total KHCL-32-R2 score in the MDD group $(12.73 \pm 8.21)$ was significantly lower than that in the BD (18.82 $\pm 6.81 ; p<0.001)$, BD-I $(18.89 \pm 6.86 ; p<0.001)$, and BD-II $(18.64 \pm 6.71 ; p<0.001)$ groups; however, there was no significant difference between the BD-I and BD-II groups $(p=0.97)$ (Table 1).

The mean score for factor 1 in the MDD group (6.48 \pm 4.80) was significantly lower than that of the BD-I ( $10.23 \pm$ $4.13 ; p<0.001)$ and BD-II groups $(10.19 \pm 4.35 ; p<$ $0.001)$; however, the difference between BD-I and BD-II groups ( $p=0.95$ ) was not significant. The mean score for factor 2 in the MDD group (3.78 \pm 2.77$)$ was significantly lower than that of the BD-I $(5.51 \pm 2.61 ; p<0.001)$ and BD-II groups $(5.28 \pm 2.73 ; p<0.001)$; however, there was no significant difference between the BD-I and BD-II groups $(p=0.54)$. Finally, the mean score for factor 3 in the MDD group (2.47 \pm 2.51 ) was significantly lower than that of the BD-I $(3.16 \pm 2.53 ; p<0.01)$ and BD-II groups (3.17 $\pm 2.32 ; p<0.05)$; however, there was no significant difference between the BD-I and BD-II groups $(p=0.98)$.

\section{ROC Curve Analysis}

\section{$M D D$ vs. BD}

ROC curve analysis demonstrated that the total KHCL32-R2 score differed between MDD and BD patients ( $p<$ $0.001)$; the AUC was 0.72 (95\% CI, 0.67-0.77). A cut-off score of 16 was optimal for maximizing sensitivity $(0.70)$ and specificity (0.70) (Fig. 3A). The sensitivity and specificity for a cut-off value of 14 were 0.77 and 0.59 , respectively.

\section{$M D D$ vs. $B D-I$}

ROC curve analysis showed that KHCL-32-R2 score differed between MDD and BD-I patients $(p<0.001)$; the AUC was 0.72 (95\% CI, 0.67-0.77). A cut-off score of 16 was optimal to maximize sensitivity (0.73) and specificity (0.70) (Fig. 3B). The sensitivity and specificity of a cut-off value of 14 were 0.79 and 0.59 , respectively.

MDD vs. BD-II

ROC curve analysis revealed that the KHCL-32-R2 dif- ferentiated between MDD and BD-II patients $(p<0.001)$. The AUC was 0.71 (95\% CI, 0.65-0.78). A screening score of 15 was the optimal cut-off to maximize sensitivity (0.67) and specificity (0.67) (Fig. 3C). The sensitivity and specificity of a cut-off score of 14 were 0.72 and 0.59 , respectively.

BD-I vs. BD-II

The KHCL-32-R2 did not differentiate between BD-I and BD-II in ROC curve analysis $(p=0.81)$. The AUC was 0.51 (95\% CI, 0.43-0.59).

\section{Positive Predictive Value (PPV) and Negative Predictive Value (NPV)}

The PPV and the NPV were 0.76 and 0.63 , respectively, between MDD and BD at a cut-off of 16 according to the KHCL-32-R2.

\section{Test-retest Reliability}

A total of 158 subjects (MDD, $n=72$; BD-I, $n=70$; and BD-II, $\mathrm{n}=16$ ) completed the second KHCL-32-R2 within four weeks after the first trial. Test-retest reliability coefficient was $0.84(p<0.001)$; however, mean scores for the second KHCL-32-R2 among MDD (15.64 \pm 9.50$)$, BD-I (17.31 \pm 7.64$)$ and BD-II patients (18.63 \pm 7.17$)$ were not significantly different according to the Kruskal-Wallis test $\left(\chi^{2}=2.10, p=0.35\right)$.

\section{DISCUSSION}

The principal objective of this study was to examine the psychometric properties of the Korean adaptation of HCL-32-R2 as a screening tool for BD in mood disorder patients. Compared to patients with MDD, patients with $\mathrm{BD}$ were younger and had a younger age at onset and more frequent episodes, which was consistent with previously reported demographic and clinical features of $\mathrm{BD}$ patients. ${ }^{2,12)}$ Although the patients with BD were more educated, due to the clinical features of $\mathrm{BD}$, they may be at increased risk for relationship problems, which may explain why fewer BD patients in present study were married. ${ }^{24,25)}$

The optimal cut-off value between MDD and BD was 16; similarly, the optimal cut-off value between MDD and BD-I was also 16, while the optimal cut-off value between MDD and BD-II was 15 . These cut-off values were slightly higher than those reported in a large transcultural study including 18 countries (Europe, North Africa, Near East, Far East), ${ }^{13)}$ an Italian study ${ }^{23,26)}$ and in studies from 
Russia $^{27)}$ and China, ${ }^{28)}$ all of which used the HCL-32-R2, and furthermore in a Korean study using the HCL-32R1. ${ }^{14)}$ At present, there is no obvious pattern (e.g., setting, sample, or cultural background) that would account for the variance in the optimal cut-off value. ${ }^{29)}$ Our findings that the HCL-32-R2 does not distinguish between BD-I and $\mathrm{BD}-\mathrm{II}$ is consistent with results from previous studies testing the Italian, Arabic and Chinese versions of this instrument, ${ }^{23,26,30)}$ with some exceptions. ${ }^{31,32)}$ By changing the 4-day duration for hypomania to 2 days, the HCL-32 $\mathrm{R} 2$ can distinguish between BD-I and BD-II with an optimal cut-off value of $14 .^{31)}$ Another explanation for differentiation may be the considerable difference in the proportion of MDD, BD-I and BD-II and the episode status (sample-specific prevalence). ${ }^{29)}$

The value of Cronbach's alpha for the KHCL-32-R2 was 0.91 , which was slightly higher than the previous studies. $^{23,26,29)}$ The internal consistency of HCL-32 reported by previous studies has been excellent ${ }^{29)}$ and results from the present study are also consistent with these previous results.

The factor structure in the present study is somewhat different from previous studies that administered the HCL-32-R1 and R2. ${ }^{4,23,26,30)}$ Most studies have favored two-factor solutions, as suggested in the original study, which reflected a bright and dark side of hypomania; however, these studies also did not reject the possibility of there being three-factor or greater structures for HCL-32. ${ }^{23,24)}$ A previous Korean study outlined a threefactor structure for HCL-32-R1, determining the three factors to be "elated mood/increased energy," "risk-taking behavior/irritability" and "increased sexual activity". ${ }^{15)}$ This study also identified a three-factor structure through exploratory factor analysis, which could be labeled as "active/elated," "irritable/distractible" and "risk-taking/ indulging." Of the HCL-32-R2 items, those regarding sexual activity, drinking, eating and other activities were classified as the unique dimension in this study that was outside the realm of previous studies. ${ }^{4,15,29)}$ Further studies may be required to determine whether this dimension can be considered a distinct factor.

As with results from previous studies using HCL$32,{ }^{4,24,31)}$ there were no significant relationships between the HCL-32-R2 score and the severity of depression or manic symptoms measured with MADRS and YMRS. The HCL-32-R2 is highly correlated with MDQ scores $(\mathrm{r}=0.73)$ and test-retest reliability is also good $(\mathrm{r}=0.84)$, which suggests its psychometric properties are robust.

Some limitations of this study should be acknowledged.
Although the study used a multi-center design and inclusion of in- and out-patients sample was used to avoid sample-specific prevalence of $\mathrm{BD}$, nevertheless, there may be a selection bias due to non-equivalent proportions of BD-I and BD-II. Although the inclusion of BD-NOS was permitted in this study, there were no patients with that diagnosis, and hence it may limit the conclusions that can be drawn from this study. Secondly, psychiatric co-morbidity was not measured, which could have influenced the sensitivity and specificity of the HCL-32R2. ${ }^{24,29)}$ Finally, we did not further analyze with more recent statistical methods such as confirmatory factor analysis or Item Response Theory. ${ }^{24,25)}$ Further studies will be needed to verify the strength of the KHCL-32-R2 as the screening tools for BDs from MDD in the context of recent literature.

The results of this study indicate that the psychometric properties of the Korean adaptation of the HCL-32-R2 are satisfactory when tested in mood disorder patients. We expect the Korean adaptation of the HCL-32-R2 to be useful as a screening tool for BD in clinical practice.

\section{- Acknowledgments}

This study was partially funded by Pfizer Pharmaceutical. The company had no role in the study design; in the collection, analysis and interpretation of data; in the writing of the report; and in the decision to submit the paper for publication.

\section{REFERENCES}

1. Muneer A. Staging models in bipolar disorder: A systematic review of the literature. Clin Psychopharmacol Neurosci 2016;14:117-130.

2. Carta MG, Angst J. Epidemiological and clinical aspects of bipolar disorders: controversies or a common need to redefine the aims and methodological aspects of surveys. Clin Pract Epidemiol Ment Health 2005;1:4.

3. Hirschfeld RM, Lewis L, Vornik LA. Perceptions and impact of bipolar disorder: how far have we really come? Results of the national depressive and manic-depressive association 2000 survey of individuals with bipolar disorder. J Clin Psychiatry 2003;64:161-174.

4. Angst J, Adolfsson R, Benazzi F, Gamma A, Hantouche E, Meyer TD, et al. The HCL-32: towards a self-assessment tool for hypomanic symptoms in outpatients. J Affect Disord 2005;88:217-233.

5. Carvalho AF, Takwoingi Y, Sales PM, Soczynska JK, Köhler CA, Freitas TH, et al. Screening for bipolar spectrum disorders: A comprehensive meta-analysis of accuracy studies. J Affect Disord 2015;172:337-346.

6. Muneer A. Pharmacotherapy of bipolar disorder with quetiapine: A recent literature review and an update. Clin Psychopharmacol Neurosci 2015;13:25-35.

7. Angst J, Ajdacic-Gross V, Rössler W. Classification of 
mood disorders. Psychiatr Pol 2015;49:663-671.

8. Sheehan DV, Lecrubier Y, Sheehan KH, Amorim P, Janavs $\mathrm{J}$, Weiller E, et al. The Mini-International Neuropsychiatric Interview (M.I.N.I.): the development and validation of a structured diagnostic psychiatric interview for DSM-IV and ICD-10. J Clin Psychiatry 1998;59 Suppl 20:22-33;quiz 34-57.

9. First MB, Spitzer RL, Gibbon M, Williams JBW. Structured clinical interview for DSM-IV axis I disorders, clinician version (SCID-CV). Washington, D.C.:American Psychiatric Press, Inc.; 1996.

10. Hirschfeld RM, Williams JB, Spitzer RL, Calabrese JR, Flynn L, Keck PE Jr, et al. Development and validation of a screening instrument for bipolar spectrum disorder: the Mood Disorder Questionnaire. Am J Psychiatry 2000;157: 1873-1875.

11. Nassir Ghaemi S, Miller CJ, Berv DA, Klugman J, Rosenquist KJ, Pies RW. Sensitivity and specificity of a new bipolar spectrum diagnostic scale. J Affect Disord 2005;84: 273-277.

12. Angst J, Azorin JM, Bowden CL, Perugi G, Vieta E, Gamma A, et al. Prevalence and characteristics of undiagnosed bipolar disorders in patients with a major depressive episode: the BRIDGE study. Arch Gen Psychiatry 2011;68: 791-798.

13. Gamma A, Angst J, Azorin JM, Bowden CL, Perugi G, Vieta E, et al. Transcultural validity of the Hypomania Checklist-32 (HCL-32) in patients with major depressive episodes. Bipolar Disord 2013;15:701-712.

14. Oh MY, Angst J, Sung TH, Lee EH, Hong KS, Lee DS, et al. Reliability and validity of the Hypomania Symptom Checklist-32 in Korea. Korean J Clin Psychol 2009;28: 321-338.

15. An D, Hong KS, Kim JH. Exploratory factor analysis and confirmatory factor analysis of the Korean version of Hypomania Checklist-32. Psychiatry Investig 2011;8:334339.

16. Montgomery SA, Asberg M. A new depression scale designed to be sensitive to change. Br J Psychiatry 1979; 134:382-389.

17. Ahn YM, Lee KY, Yi JS, Kang MH, Kim DH, Kim JL, et al. A validation study of the Korean-version of the Montgomery-Asberg Depression Rating Scale. J Korean Neuropsychiatr Assoc 2005;44:466-476.

18. Young RC, Biggs JT, Ziegler VE, Meyer DA. A rating scale for mania: reliability, validity and sensitivity. Br J Psychiatry 1978; 133:429-435.

19. Jung HY, Cho HS, Joo YH, Shin HK, Yi JS, Hwang S, et al. A validation study of the Korean-version of the young mania rating scale. J Korean Neuropsychiatr Assoc 2003; 42:263-269.

20. Jon DI, Hong N, Yoon BH, Jung HY, Ha K, Shin YC, et al. Validity and reliability of the Korean version of the Mood Disorder Questionnaire. Compr Psychiatry 2009;50: 286-291.

21. Angst J, Meyer TD, Adolfsson R, Skeppar P, Carta M, Benazzi F, et al. Hypomania: a transcultural perspective. World Psychiatry 2010;9:41-49.

22. Portney LG, Watkins MP. Foundations of clinical research: applications to practice. Upper Saddle River, NJ:Prentice Hall Health;2000.

23. Fornaro M, De Berardis D, Mazza M, Pino M, Favaretto E, Bedani $\mathrm{F}$, et al. Factor structure and reliability of the Italian adaptation of the Hypomania Check List-32, second revision (HCL-32-R2). J Affect Disord 2015;178:112-120.

24. Feng Y, Xiang YT, Huang W, Wang G, Feng L, Tian TF, et al. The 33-item Hypomania Checklist (HCL-33): A new self-completed screening instrument for bipolar disorder. $J$ Affect Disord 2016;190:214-220.

25. Mitchell PB, Goodwin GM, Johnson GF, Hirschfeld RM. Diagnostic guidelines for bipolar depression: a probabilistic approach. Bipolar Disord 2008;10:144-152.

26. Fornaro M, Elassy M, Mounir M, Abd-Elmoneim N, Ashour $\mathrm{H}$, Hamed $\mathrm{R}$, et al. Factor structure and reliability of the Arabic adaptation of the Hypomania Check List-32, second revision (HCL-32-R2). Compr Psychiatry 2015;59:141-150.

27. Mosolov SN, Ushkalova AV, Kostukova EG, Shafarenko $\mathrm{AA}$, Alfimov PV, Kostyukova $\mathrm{AB}$, et al. Validation of the Russian version of the Hypomania Checklist (HCL-32) for the detection of bipolar II disorder in patients with a current diagnosis of recurrent depression. $J$ Affect Disord 2014; 155:90-95.

28. Yang HC, Xiang YT, Liu TB, Han R, Wang G, Hu C, et al. Hypomanic symptoms assessed by the HCL-32 in patients with major depressive disorder: a multicenter trial across China. J Affect Disord 2012;143:203-207.

29. Meyer TD, Schrader J, Ridley M, Lex C. The Hypomania Checklist (HCL) - systematic review of its properties to screen for bipolar disorders. Compr Psychiatry 2014;55: 1310-1321.

30. Yang HC, Yuan CM, Liu TB, Li LJ, Peng HJ, Liao CP, et al. Validity of the 32-item Hypomania Checklist (HCL-32) in a clinical sample with mood disorders in China. BMC Psychiatry 2011;11:84.

31. Wu YS, Angst J, Ou CS, Chen HC, Lu RB. Validation of the Chinese version of the hypomania checklist (HCL-32) as an instrument for detecting hypo(mania) in patients with mood disorders. J Affect Disord 2008;106:133-143.

32. Vieta E, Sánchez-Moreno J, Bulbena A, Chamorro L, Ramos $\mathrm{JL}$, Artal J, et al. Cross validation with the mood disorder questionnaire $(M D Q)$ of an instrument for the detection of hypomania in Spanish: the 32 item hypomania symptom check list (HCL-32). J Affect Disord 2007;101:43-55. 